\title{
H.E.S.S. detection of very high-energy $\gamma$-ray emission from the quasar PKS 0736+017
}

H.E.S.S. Collaboration: H. Abdalla ${ }^{1}$, R. Adam ${ }^{2}$, F. Aharonian ${ }^{3,4,5}$, F. Ait Benkhali ${ }^{3}$, E. O. Angüner ${ }^{6}$, M. Arakawa ${ }^{7}$, C. Arcaro ${ }^{1}$, C. Armand ${ }^{8}$, H. Ashkar ${ }^{9}$, M. Backes ${ }^{10,1}$, V. Barbosa Martins ${ }^{11}$, M. Barnard ${ }^{1}$, Y. Becherini ${ }^{12}$, D. Berge ${ }^{11}$, K. Bernlöhr ${ }^{3}$, R. Blackwell ${ }^{13}$, M. Böttcher $^{1}$, C. Boisson ${ }^{14}$, J. Bolmont ${ }^{15}$, S. Bonnefoy ${ }^{11}$, J. Bregeon ${ }^{16}$, M. Breuhaus ${ }^{3}$, F. Brun ${ }^{9}$, P. Brun ${ }^{9}$, M. Bryan ${ }^{17}$, M. Büchele ${ }^{18}$, T. Bulik ${ }^{19}$, T. Bylund ${ }^{12}$, M. Capasso ${ }^{20}$, S. Caroff ${ }^{15}$, A. Carosi ${ }^{8}$, S. Casanova ${ }^{21,3}$, M. Cerruti ${ }^{15,42, \star}$, T. Chand ${ }^{1}$, S. Chandra $^{1}$, A. Chen ${ }^{22}$, S. Colafrancesco $^{22, \dagger}$, M. Curyło $^{23}$, I. D. Davids ${ }^{10}$, C. Deil ${ }^{3}$, J. Devin ${ }^{24}$, P. deWilt ${ }^{13}$, L. Dirson ${ }^{25}$, A. Djannati-Atai ${ }^{26}$, A. Dmytriiev ${ }^{14}$, A. Donath ${ }^{3}$, V. Doroshenko ${ }^{20}$, L. O'C. Drury ${ }^{4}$, J. Dyks ${ }^{27}$, K. Egberts ${ }^{28}$, G. Emery ${ }^{15}$, J.-P. Ernenwein ${ }^{6}$, S. Eschbach $^{18}$, K. Feijen ${ }^{13}$, S. Fegan ${ }^{2}$, A. Fiasson ${ }^{8}$, G. Fontaine ${ }^{2}$, S. Funk ${ }^{18}$, M. Füßling ${ }^{11}$, S. Gabici ${ }^{26}$, Y. A. Gallant ${ }^{16}$, F. Gaté ${ }^{8}$, G. Giavitto ${ }^{11}$, D. Glawion ${ }^{29}$, J. F. Glicenstein ${ }^{9}$, D. Gottschall ${ }^{20}$, M.-H. Grondin $^{24}$, J. Hahn $^{3}$, M. Haupt ${ }^{11}$, G. Heinzelmann ${ }^{25}$, G. Henri ${ }^{30}$, G. Hermann ${ }^{3}$, J. A. Hinton ${ }^{3}$, W. Hofmann ${ }^{3}$, C. Hoischen ${ }^{28}$, T. L. Holch ${ }^{31}$, M. Holler ${ }^{32}$, D. Horns ${ }^{25}$, D. Huber ${ }^{32}$, H. Iwasaki ${ }^{7}$, M. Jamrozy ${ }^{23}$, D. Jankowsky ${ }^{18}$, F. Jankowsky ${ }^{29}$, A. Jardin-Blicq ${ }^{3}$, I. Jung-Richardt ${ }^{18}$, M. A. Kastendieck ${ }^{25}$, K. Katarzyński ${ }^{33}$, M. Katsuragawa ${ }^{34}$, U. Katz ${ }^{18}$, D. Khangulyan ${ }^{7}$, B. Khélififi ${ }^{26}$, J. King ${ }^{29}$, S. Klepser ${ }^{11}$, W. Kluźniak ${ }^{27}, N_{u}$. Kominn ${ }^{22}$, K. Kosack ${ }^{9}$, D. Kostunin ${ }^{11}$, M. Kraus ${ }^{18}$, G. Lamanna ${ }^{8}$, J. Lau ${ }^{13}$, A. Lemière ${ }^{26}$, M. Lemoine-Goumard ${ }^{24}$, J.-P. Lenain ${ }^{15, \star}$, E. Leser ${ }^{28,11}$, C. Levy ${ }^{15}$, T. Lohse $^{31}$, I. Lypova ${ }^{11}$, J. Mackey ${ }^{4}$, J. Majumdar ${ }^{11}$, D. Malyshev ${ }^{20}$, V. Marandon ${ }^{3}$, A. Marcowith ${ }^{16}$, A. Mares ${ }^{24}$, C. Mariaud ${ }^{2}$, G. Martí-Devesa ${ }^{32}$, R. Marx ${ }^{3}$, G. Maurin ${ }^{8}$, P. J. Meintjes ${ }^{35}$, A. M. W. Mitchell ${ }^{3,43}$, R. Moderski ${ }^{27}$, M. Mohamed ${ }^{29}$, L. Mohrmann $^{18}$, J. Muller $^{2}$, C. Moore ${ }^{36}$, E. Moulin ${ }^{9}$, T. Murach ${ }^{11}$, S. Nakashima ${ }^{37}$, M. de Naurois ${ }^{2}$, H. Ndiyavala ${ }^{1}$, F. Niederwanger ${ }^{32}$, J. Niemiec ${ }^{21}$, L. Oakes $^{31}$, P. O’Brien ${ }^{36}$, H. Odaka ${ }^{38}$, S. Ohm ${ }^{11}$, E. de Oña Wilhelmi ${ }^{11}$, M. Ostrowski ${ }^{23}$, I. Oya ${ }^{11}$, M. Panter ${ }^{3}$, R. D. Parsons ${ }^{3}$, C. Perennes ${ }^{15}$, P.-O. Petrucci ${ }^{30}$, B. Peyaud ${ }^{9}$, Q. Piel ${ }^{8}$, S. Pita ${ }^{26}$, V. Poireau ${ }^{8}$, A. Priyana Noel ${ }^{23}$, D. A. Prokhorov ${ }^{22}$, H. Prokoph ${ }^{11, \star}$, G. Pühlhofer ${ }^{20}$, M. Punch ${ }^{26,12}$, A. Quirrenbach ${ }^{29}$, S. Raab ${ }^{18}$, R. Rauth ${ }^{32}$, A. Reimer ${ }^{32}$, O. Reimer ${ }^{32}$, Q. Remy ${ }^{16}$, M. Renaud ${ }^{16}$, F. Rieger $^{3}$, L. Rinchiuso ${ }^{9}$, C. Romoli ${ }^{3}$, G. Rowell ${ }^{13}$, B. Rudak ${ }^{27}$, E. Ruiz-Velasco ${ }^{3}$, V. Sahakian ${ }^{39}$, S. Saito ${ }^{7}$, D. A. Sanchez ${ }^{8}$, A. Santangelo ${ }^{20}$, M. Sasaki $^{18}$, R. Schlickeiser ${ }^{40}$, F. Schüssler ${ }^{9}$, A. Schulz ${ }^{11}$, H. Schutte ${ }^{1}$, U. Schwanke ${ }^{31}$, S. Schwemmer ${ }^{29}$, M. Seglar-Arroyo ${ }^{9}$, M. Senniappan ${ }^{12}$, A. S. Seyffert ${ }^{1}$, N. Shafi ${ }^{22}$, K. Shiningayamwe ${ }^{10}$, R. Simoni ${ }^{17}$, A. Sinha ${ }^{26}$, H. Sol $^{14}$, A. Specovius ${ }^{18}$, M. Spir-Jacob ${ }^{26}$, Ł. Stawarz $^{23}$, R. Steenkamp ${ }^{10}$, C. Stegmann ${ }^{28,11}$, C. Steppa ${ }^{28}$, T. Takahashi ${ }^{34}$, T. Tavernier ${ }^{9}$, A. M. Taylor ${ }^{11}$, R. Terrier ${ }^{26}$, D. Tiziani ${ }^{18}$, M. Tluczykont $^{25}$, C. Trichard ${ }^{2}$, M. Tsirou ${ }^{16}$, N. Tsuji ${ }^{7}$, R. Tuffs ${ }^{3}$, Y. Uchiyama ${ }^{7}$, D. J. van der Walt ${ }^{1}$, C. van Eldik ${ }^{18}$, C. van Rensburg ${ }^{1}$, B. van Soelen $^{35}$, G. Vasileiadis ${ }^{16}$, J. Veh ${ }^{18}$, C. Venter ${ }^{1}$, P. Vincent ${ }^{15}$, J. Vink ${ }^{17}$, F. Voisin ${ }^{13}$, H. J. Völk ${ }^{3}$, T. Vuillaume ${ }^{8}$, Z. Wadiasingh ${ }^{1}$, S. J. Wagner ${ }^{29}$, R. White ${ }^{3}$, A. Wierzcholska ${ }^{21,29}$, R. Yang ${ }^{3}$, H. Yoneda ${ }^{34}$, M. Zacharias ${ }^{1}$, R. Zanin ${ }^{3}$, A. A. Zdziarski ${ }^{27}$, A. Zech ${ }^{14}$, A. Ziegler $^{18}$, J. Zorn $^{3}$, N. Żywucka ${ }^{1}$, and P. S. Smith ${ }^{41}$

(Affiliations can be found after the references)

Received 13 May 2019 / Accepted 12 November 2019

\section{ABSTRACT}

Context. Flat-spectrum radio-quasars (FSRQs) are rarely detected at very high energies $(E \geq 100 \mathrm{GeV})$ due to their low-frequency-peaked spectral energy distributions. At present, only six FSRQs are known to emit very high-energy (VHE) photons, representing only 7\% of the VHE extragalactic catalog, which is largely dominated by high-frequency-peaked BL Lacertae objects.

Aims. Following the detection of MeV-GeV $\gamma$-ray flaring activity from the FSRQ PKS $0736+017(z=0.189)$ with Fermi-LAT, the H.E.S.S. array of Cherenkov telescopes triggered target-of-opportunity (ToO) observations on February 18, 2015, with the goal of studying the $\gamma$-ray emission in the VHE band.

Methods. H.E.S.S. ToO observations were carried out during the nights of February 18, 19, 21, and 24, 2015. Together with Fermi-LAT, the multi-wavelength coverage of the flare includes Swift observations in soft X-ray and optical-UV bands, and optical monitoring (photometry and spectro-polarimetry) by the Steward Observatory, and the ATOM, the KAIT, and the ASAS-SN telescopes.

Results. VHE emission from PKS 0736+017 was detected with H.E.S.S. only during the night of February 19, 2015. Fermi-LAT data indicate the presence of a $\gamma$-ray flare, peaking at the time of the H.E.S.S. detection, with a flux doubling timescale of around six hours. The $\gamma$-ray flare was accompanied by at least a 1 mag brightening of the non-thermal optical continuum. No simultaneous observations at longer wavelengths are available for the night of the H.E.S.S. detection. The $\gamma$-ray observations with H.E.S.S. and Fermi-LAT are used to put constraints on the location of the $\gamma$-ray emitting region during the flare: it is constrained to be just outside the radius of the broad-line region $r_{B L R}$ with a bulk Lorentz factor $\Gamma \simeq 20$, or at the level of the radius of the dusty torus $r_{\text {torus }}$ with $\Gamma \simeq 60$.

Conclusions. PKS $0736+017$ is the seventh FSRQ known to emit VHE photons, and at $z=0.189$ is the nearest so far. The location of the $\gamma$-ray emitting region during the flare can be tightly constrained thanks to opacity, variability, and collimation arguments.

Key words. gamma rays: general - astroparticle physics - relativistic processes - quasars: individual: PKS 0736+017

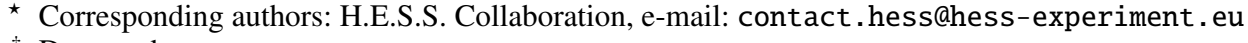

$\dagger$ Deceased.
} 


\section{Introduction}

The very high-energy (VHE; $E \geq 100 \mathrm{GeV}$ ) window on the Universe was opened with the discovery of VHE emission from the Crab Nebula (Weekes et al. 1989) using the Whipple 10-m Imaging Atmospheric Cherenkov Telescope (IACT). Soon after, the first extragalactic VHE source, the blazar Markarian 421, was discovered by Punch et al. (1992). A few decades later, thanks to the current generation of IACTs (H.E.S.S., MAGIC, and VERITAS), the number of known VHE extragalactic sources has grown to $82^{1}$. The majority of them (around 90\%) are blazars.

Within the current unification model of active galactic nuclei (AGNs), a blazar is interpreted as a radio-loud AGN whose relativistic jet points in the direction of the observer (see Blandford $\&$ Rees 1978). From an observational point of view, two subclasses of blazars exist, flat-spectrum radio-quasars (FSRQs) and BL Lacertae objects, according to the equivalent width of emission lines from the broad-line region (BLR), which is $>5 \AA$ in FSRQs (see, e.g., Urry \& Padovani 1995). All blazars are characterized by a similar spectral energy distribution (SED), which consists of two distinct components peaking in the infrared to $\mathrm{X}$-ray band and in the MeV-TeV band, respectively (see, e.g., Abdo et al. 2010). While FSRQs are in general characterized by a relatively low frequency (in the infrared) of the low-energy SED peak, BL Lac objects are further classified by the peak frequency of their first SED component into low-, intermediate-, and high-frequency-peaked BL Lac objects (LBLs, IBLs, and HBLs; Padovani \& Giommi 1995; Sambruna et al. 1996). Hence, observations in a narrow frequency window unavoidably preselect a particular blazar subclass, and it is not a surprise that observations at VHE $\gamma$-rays detect more likely HBLs whose overall spectrum can peak in the VHE band at energies up to few TeVs (such as the extreme HBL 1ES 0229+200; see Aharonian et al. 2007; Aliu et al. 2014), and represent about $70 \%$ of extragalactic VHE sources. In addition, FSRQs and BL Lac objects have different redshift distributions, with the former being located on average at larger distances (see Padovani 1992). VHE astronomy has the important property of being limited in redshift, due to the absorption of VHE photons via pair-production over the extragalactic background light (EBL; see Salamon \& Stecker 1998). Thus, both intrinsic source properties and propagation effects make FSRQs difficult to be observed with IACTs. So far only six FSRQs have been detected by IACTs: 3C 279 (MAGIC Collaboration 2008; H.E.S.S. Collaboration 2019), PKS 1222+ 216 (Aleksić et al. 2011; Cerruti 2015); PKS 1510-089 (H.E.S.S. Collaboration 2013; Aleksić et al. 2014); PKS 1441+252 (Abeysekara et al. 2015; Ahnen et al. 2015); S3 0218+35 (Ahnen et al. 2016), which is currently the most distant source of VHE photons ever observed $(z=0.944)$; and Ton 599 (Mirzoyan 2017; Mukherjee 2017). With the notable exception of PKS 1510-089 (MAGIC Collaboration 2018), which at $z=0.361$ is the nearest, all the other FSRQs have been detected at VHE only during bright flaring activity, and are all characterized by very soft VHE spectra.

The radiation mechanism responsible for the low-energy SED component of blazars is thought to be synchrotron emission by a non-thermal population of leptons (electrons and positrons) in the jet. The radiation mechanism responsible for the $\gamma$-ray emission is thought to be inverse-Compton scattering off lowenergy photons by the same leptons that produce the synchrotron SED component. For FSRQs, the low-energy target photons are thought to be thermal photons from the accretion disk, or from the dusty torus, or emission lines produced in the BLR (see

\footnotetext{
1 In October 2019. For an up-to-date list of VHE sources see http: //tevcat.uchicago . edu, Wakely \& Horan (2008).
}

Dermer \& Schlickeiser 1993; Sikora et al. 1994; Błażejowski et al. 2000). This type of scenario, called external inverseCompton (EIC), proved to be able to successfully reproduce the broad-band SED of $\gamma$-ray FSRQs (see, e.g., Ghisellini et al. 2010; Meyer et al. 2012; Böttcher et al. 2013). Alternative hadronic emission scenarios, although capable of modeling the SED of FSRQs (see, e.g., Böttcher et al. 2013), encounter difficulties due to the high power required to reproduce the photon emission (Sikora et al. 2009; Reimer 2012; Petropoulou \& Dimitrakoudis 2015; Zdziarski \& Böttcher 2015).

Even though there is general consensus on the EIC as the emission mechanism, several questions remain open. Among them is the uncertainty as to where the $\gamma$-ray emission region is located within the relativistic jet. Does the emission come from the base of the jet, near the supermassive black hole (SMBH) powering the quasar, or is it instead produced downstream in the jet? The answer to this question, which arose with the detection of the first $\gamma$-ray AGNs by EGRET (see, e.g., Dermer et al. 1992; Dermer \& Schlickeiser 1994; Becker \& Kafatos 1995; Blandford \& Levinson 1995; Marcowith et al. 1995; Jorstad et al. 2001), is still a major active research topic in blazar physics and has been addressed by several authors in recent years (see, e.g., Ghisellini \& Tavecchio 2009; Finke \& Dermer 2010; Poutanen \& Stern 2010; Tavecchio et al. 2010; Agudo et al. 2011a,b; Hayashida et al. 2012; Yan et al. 2012; Cao \& Wang 2013; Rani et al. 2013; Brown 2013; Dermer et al. 2014; Max-Moerbeck et al. 2014; Nalewajko et al. 2014; Dotson et al. 2015; Ramakrishnan et al. 2015; Coogan et al. 2016; Finke 2016). In this paper we show that by making use only of $\gamma$-ray observations of the quasar PKS $0736+017$, it is possible to put a tight constraint on the location of the $\gamma$-ray emitting region within the jet.

The quasar PKS 0736+017 was first detected as a radio-source with the Parkes telescope (Day et al. 1966). Its radio morphology is typical of a blazar, with a compact core and a single-sided, parsec-scale jet (Lister \& Homan 2005; Lister et al. 2009). The optical-UV spectrum is characterized by broad emission lines, and a big blue bump that is associated with thermal emission from the SMBH accretion disk (Baldwin 1975; Malkan \& Moore 1986). Thanks to the emission lines, the redshift of PKS $0736+017$ is well determined: the most recent measurement is $z=0.189$ (Ho \& Kim 2009). As is typical for blazars, a giant elliptical galaxy hosts the AGN (Kotilainen et al. 1998; Wright et al. 1998; McLure et al. 1999). During January 2002, PKS 0736+017 exhibited an extremely bright and fast optical flare $\left(0.6 \mathrm{mag} \mathrm{h}^{-1}\right.$, see Clements et al. 2003), which also classifies the source as an Optically Violently Variable (OVV) quasar.

In high-energy $\gamma$-rays (HE; $100 \mathrm{MeV} \leq E \leq 100 \mathrm{GeV}$ ), PKS $0736+017$ is detected by Fermi-LAT, and is included in the most recent Fermi-LAT catalog (3FGL; Acero et al. 2015) under the name $3 F G L J 0739.4+0137$. Since the beginning of the Fermi mission in 2008, the source remained relatively quiescent until November 2014, when a $\gamma$-ray flare was detected (D'Ammando \& Orienti 2014). The source remained active in HE $\gamma$-rays for the following months, and reached its maximum $\gamma$-ray flux in February 2015. The flaring state in HE $\gamma$-rays triggered VHE observations with the High Energy Stereoscopic System (H.E.S.S.), resulting in the first detection of VHE photons from PKS 0736+017, which is reported in this paper.

The paper is organized as follows: in Sect. 2 we present the observations of PKS $0736+017$ and the data analysis, for H.E.S.S. and for lower-energy instruments; in Sect. 3 we discuss the implications of the VHE detection, in particular in terms of the location of the $\gamma$-ray emitting region, and we present the SED of the source during the flare; we draw our conclusions in Sect. 4. 
Table 1. Details of the H.E.S.S. observations of PKS 0736+017.

\begin{tabular}{lccc}
\hline \hline Array configuration & $\begin{array}{c}\text { Starting time } \\
{[\mathrm{UTC}]}\end{array}$ & $\begin{array}{c}\text { Exposure } \\
{[\mathrm{h}]}\end{array}$ & $\begin{array}{c}\text { Flux }(E>100 \mathrm{GeV}) \\
{\left[10^{-11} \mathrm{~cm}^{-2} \mathrm{~s}^{-1}\right]}\end{array}$ \\
\hline CT1-5 & Feb. 18, 2015, 21:21 & 1.8 & $<3.6$ \\
CT1-5 & Feb. 19, 2015, 18:53 & 1.8 & $5.7 \pm 1.1$ \\
CT1-5 & Feb. 21, 2015, 20:31 & 2.7 & $<2.0$ \\
CT5 & Feb. 24, 2015, 21:43 & 0.9 & $<3.1$ \\
\hline
\end{tabular}

\section{Observations and data analysis}

\subsection{H.E.S.S}

H.E.S.S. is an array of IACTs located in the Khomas Highland of Namibia $\left(23^{\circ} 16^{\prime} 18^{\prime \prime} \mathrm{S}, 16^{\circ} 30^{\prime} 00^{\prime \prime} \mathrm{E}\right)$, at an altitude of about $1800 \mathrm{~m}$ above sea level. As all other IACTs do, H.E.S.S. images the Cherenkov light emitted by particle showers triggered by interaction of $\gamma$-rays with the Earth's atmosphere. The study of the shower images makes it possible to reconstruct the incoming direction of a $\gamma$-ray, its energy, and its arrival time. The array consists of four $12 \mathrm{~m}$ diameter reflectors arranged in a square of $120 \mathrm{~m}$ side length and, since 2012, one additional $28 \mathrm{~m}$ diameter telescope (in the following called CT5) in the middle of the array (H.E.S.S. Collaboration 2017). This hybrid configuration of the array allows data to be taken in different modes by triggering on events either detected by CT5 only (monoscopic mode) or by any combination of two or more telescopes (stereoscopic mode). The standard observation mode is to collect both monoscopic and stereoscopic events during the same observation to allow for a low-energy analysis threshold (below $100 \mathrm{GeV}$ in the monoscopic mode), as well as a good spatial and spectral reconstruction based on the excellent background rejection power in the stereoscopic mode.

Target-of-opportunity (ToO) observations of PKS 0736+017 with H.E.S.S. were triggered on February 18, 2015, following the detection of flaring activity in Fermi-LAT data (see next section). H.E.S.S. observations were carried out with the full array on the same night. Follow-up observations were performed on February 19, 21, and 24 (with the full array for the first two nights, while data were recorded with CT5 only on the last night). All observations were taken in "wobble mode" where the source position is offset by 0.5 from the camera center to allow for simultaneous background estimation (Berge et al. 2007). The details of H.E.S.S. observations are listed in Table 1.

Data were analyzed with the ImPACT analysis chain (Parsons \& Hinton 2014; Parsons et al. 2015) using both monoscopic and stereoscopic reconstruction, and were crosschecked with an independent analysis chain (De Naurois \& Rolland 2009; Holler et al. 2015) yielding consistent results. With the loose configuration of the monoscopic analysis, an excess of 473 $\gamma$-like events with a signal-to-background ratio of 0.17 is found at the source position in the overall good-quality 7.2-h dataset, corresponding to a detection significance of $8.5 \sigma$ using Eq. (17) of Li \& Ma (1983). The overall signal is dominated by the strong excess coming from the second night of observations (February 19, 2015). The monoscopic (respectively, stereoscopic) analysis of this $1.8 \mathrm{~h}$ live time dataset yields an excess of 364 (49) $\gamma$-like events, corresponding to an $11.1 \sigma(5.3 \sigma)$ post-trial $^{2}$ significance, while the source is not detected with a significance greater than $5 \sigma$ on any other night.

To determine the position of the VHE $\gamma$-ray emission, a two-dimensional fit to the $\gamma$-ray excess (for the night of

\footnotetext{
2 Four trials are considered for the computation of significances: two for the analyses (monoscopic and stereoscopic), and two for the time selection (the full dataset, and the night of February 19, 2015).
}

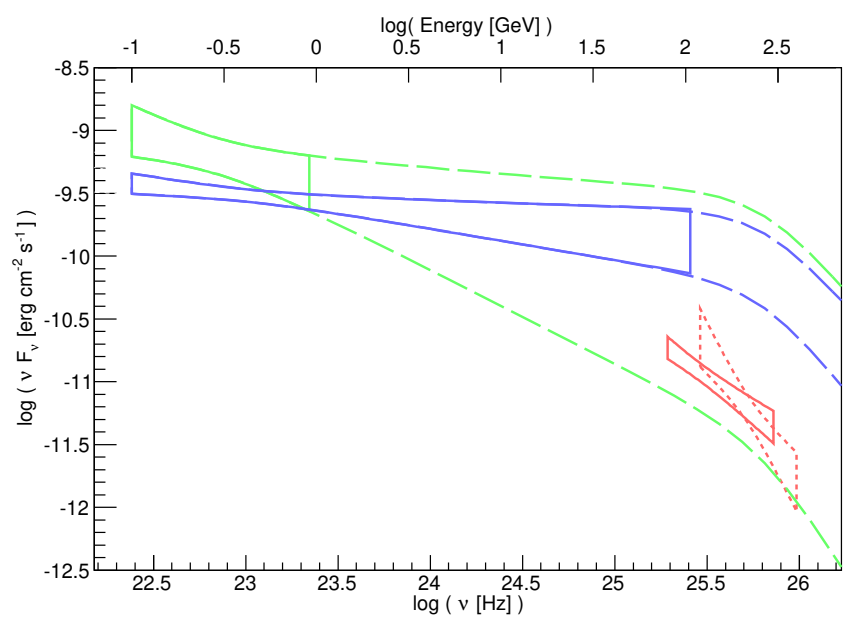

Fig. 1. $\gamma$-ray emission from PKS $0736+017$ centered on the night of February 19, 2015. The red bowtie represents the spectrum measured with H.E.S.S. using the monoscopic (bold line) and the stereoscopic analysis (dashed line). The green and blue bowties represent the spectra measured with Fermi-LAT strictly simultaneous with the H.E.S.S. detection, and integrating over an exposure of $24 \mathrm{~h}$ (MJD 57072.5-57073.5) around the H.E.S.S. detection, respectively; the extrapolation of Fermi-LAT spectra to higher energies (green and blue long-dashed lines) takes into account the absorption on the EBL (Franceschini et al. 2008).

the VHE detection only) is performed using the stereoscopic dataset, yielding a shape consistent with a point-like source. The best-fit position is found at $\mathrm{RA}(\mathrm{J} 2000)=07^{\mathrm{h}} 39^{\mathrm{m}} 17^{\mathrm{s}} \pm 3^{\mathrm{s}}$, $\operatorname{Dec}(\mathrm{J} 2000)=01^{\circ} 36^{\prime} 29^{\prime \prime} \pm 56^{\prime \prime}$, and is positionally consistent with the radio position of PKS 0736+017 (Lanyi et al. 2010).

The differential energy spectrum of the $\gamma$-ray emission is derived by performing a spectral fit, again for the night of the detection only. Both monoscopic and stereoscopic spectra are consistent with a power-law model of the form $\mathrm{d} N / \mathrm{d} E=$ $N_{0}\left(E / E_{0}\right)^{-\Gamma}$. The photon index is estimated to be $\Gamma=3.1 \pm$ $0.3_{\text {stat }} \pm 0.2_{\text {syst }}$ for the monoscopic analysis and $\Gamma=4.2 \pm$ $0.8_{\text {stat }} \pm 0.2_{\text {syst }}$ for the stereoscopic one, and the flux normalization at $200 \mathrm{GeV}$ is found to be $N_{0}=\left(1.0 \pm 0.2_{\text {stat }} \pm\right.$ $\left.0.3_{\text {syst }}\right) \times 10^{-10} \mathrm{~cm}^{-2} \mathrm{~s}^{-1} \mathrm{TeV}^{-1}$ and $N_{0}=\left(1.1 \pm 0.3_{\text {stat }} \pm 0.2_{\text {syst }}\right) \times$ $10^{-10} \mathrm{~cm}^{-2} \mathrm{~s}^{-1} \mathrm{TeV}^{-1}$, respectively. It is important to underline that the two analyses have different energy thresholds, equal to 80 and $150 \mathrm{GeV}$, respectively. The spectral results obtained are presented in Fig. 1.

The night-by-night light curve for the monoscopic reconstruction, provided as an integral flux above $100 \mathrm{GeV}$, assuming a photon index of 3.1, is shown in Fig. 2, panel a. For the nights of no detection with H.E.S.S., upper limits on the VHE emission (estimated following Rolke et al. 2005 at the 95\% confidence level) are also shown. No evidence for intra-night variability is found in the H.E.S.S. data during the night of February 19, 2015: a fit of the light curve, binned at $28 \mathrm{~min}$, with a constant function results in a $\chi^{2}$ value of 1.9 for three degrees of freedom.

\subsection{Fermi-LAT}

Detecting $\gamma$-ray photons with energies between $20 \mathrm{MeV}$ and above $300 \mathrm{GeV}$, the LAT instrument (Atwood et al. 2009) on board the Fermi satellite monitors the high-energy $\gamma$-ray sky every three hours. This instrument is thus ideal for revealing active states in AGNs, which could be used to trigger observations with other facilities as ToO observations. On February 18, 2015, an active state of this kind was detected in PKS 0736+017 

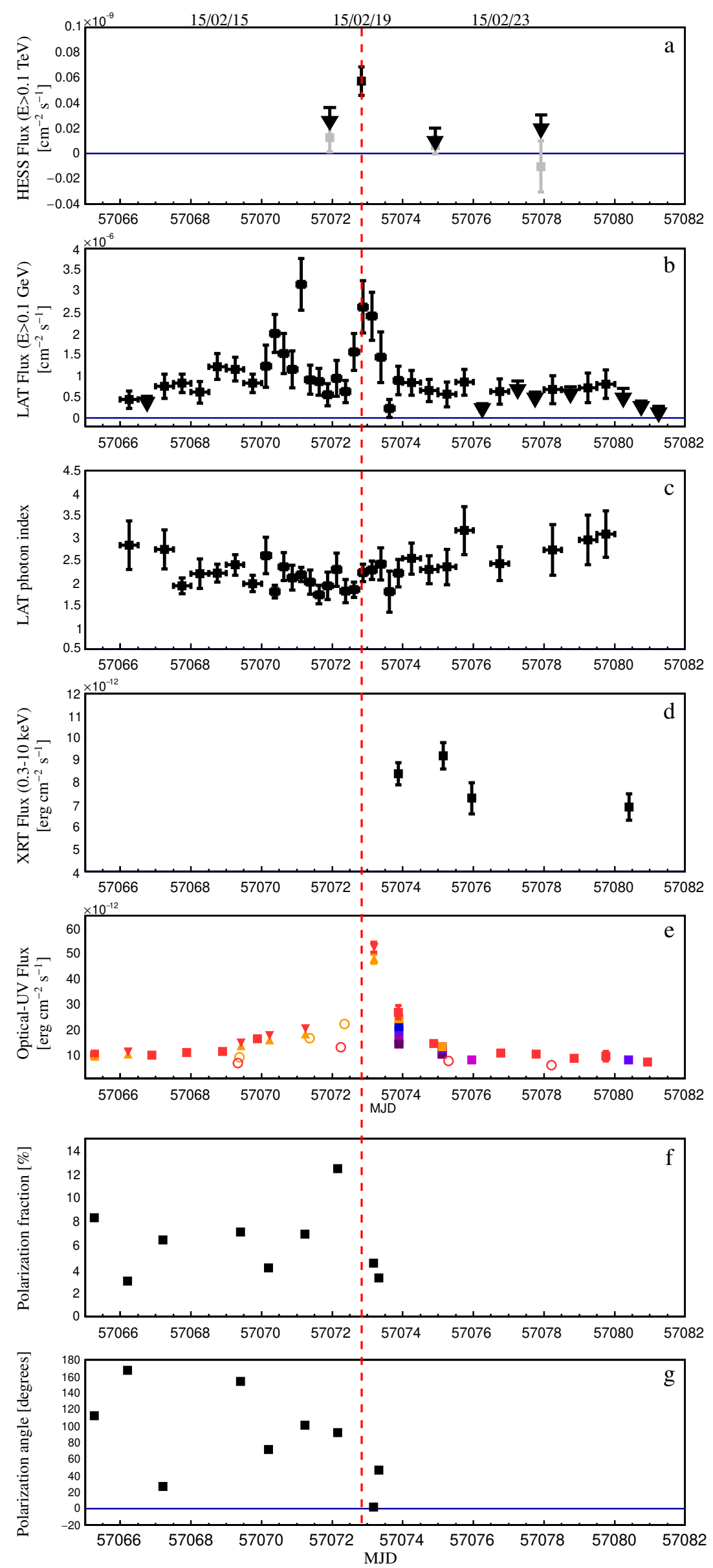

Fig. 2. Multi-wavelength light curve of PKS 0736+017 during February 2015. Panels a to g: H.E.S.S. integral flux above $100 \mathrm{GeV}$ from the monoscopic analysis (in gray, the flux for the nights with no significant detection); Fermi-LAT integral flux above $100 \mathrm{MeV}$; FermiLAT photon index; Swift-XRT integral flux between 0.3 and $10 \mathrm{keV}$, corrected for absorption; Swift-UVOT (orange, light blue, blue, light violet, violet, and dark violet squares for the $V, B, U, W 1, M 2$, and $W 2$ filter, respectively), ATOM (red squares for the $R$ filter), Steward Observatory (orange and red triangles for the $V$ and $R$ filter, respectively), ASAS-SN (orange open circles for the $V$ filter), and KAIT (red open circles, unfiltered fluxes, de-reddened); Steward Observatory broad-band polarization fraction; and Steward Observatory polarization position angle (both averaged over 5000-7000 ̊). The vertical dashed red line indicates the time of the H.E.S.S. detection. 
using the public monitored source list ${ }^{3}$ as well as the dedicated FLaapLUC aperture-photometry pipeline (Lenain 2018). Following this early flare detection, a ToO campaign was launched with H.E.S.S., as reported in the previous section.

The Fermi-LAT data are analyzed with the public ScienceTools v10rQp $5^{4}$, and events are selected within a circular region of interest of $10^{\circ}$ in radius centered on the nominal position of 3FGL J0739.4+0137 in order to perform a binned analysis as implemented in the gtlike tool. To encompass the entire active state studied here, data from between February 1 and April 1, 2015 , are considered in the $100 \mathrm{MeV}-500 \mathrm{GeV}$ energy range. The P8R3_SOURCE_V6 instrument response functions were used, together with a zenith angle cut of $90^{\circ}$ to avoid contamination by the $\gamma$-ray bright Earth limb emission. The model of the region of interest was built based on the 3FGL catalog (Acero et al. 2015), and it was checked a posteriori that no significant residual remains, which could have hinted at new sources not referenced in the 3FGL catalog. The Galactic diffuse emission was modeled using the file gll_iem_v06.fits (Acero et al. 2016) and the isotropic background using iso_P8R3_SOURCE_V6_v06.txt.

For the time window considered here, the spectrum of PKS 0736+017 is best described using a log-parabolic shape ${ }^{5}$. A comparison with a power-law spectrum yielded a log-likelihood ratio of 27.5 in favor of the log-parabola. Under this spectral hypothesis, PKS $0736+017$ is detected with a test statistic (TS; Mattox et al. 1996) of 2061 (i.e., $~ 45 \sigma$ ) with a flux $F_{(100 \mathrm{MeV}-500 \mathrm{GeV})}=(5.42 \pm 0.27) \times 10^{-7} \mathrm{~cm}^{-2} \mathrm{~s}^{-1}$, index $a=$ $2.22 \pm 0.06$, curvature index $b=0.037 \pm 0.025$, and reference energy $E_{0}=327 \mathrm{MeV}$, averaged over the two months of data considered here. In Fig. 2, panel b, the Fermi-LAT light curve of PKS 0736+017 is reported (starting from February 13, 2015), using a time binning of $6 \mathrm{~h}$ around the $\gamma$-ray flare (MJD 5707057074) and $12 \mathrm{~h}$ elsewhere, and a power-law spectrum with both the flux and photon index of PKS 0736+017 free to vary. In the light curve, flux upper limits at the $68 \%$ level are provided when $\mathrm{TS}<9$. The evolution in time of the best-fit photon index is shown in panel c.

To better compare the Fermi-LAT data of PKS 0736+017 with H.E.S.S. data during the night it was detected at VHE, and to retain sufficient statistics, a Fermi-LAT data subset was analyzed for the time window MJD 57072.5-57073.5. The source is detected in this subset with a TS $=339(\sim 18 \sigma)$, a flux of $F_{(100 \mathrm{MeV}-500 \mathrm{GeV})}=(2.08 \pm 0.26) \times 10^{-6} \mathrm{~cm}^{-2} \mathrm{~s}^{-1}$, and a photon index of $\Gamma=2.15 \pm 0.10$. In this case, no evidence of curvature is found (log-likelihood ratio of -0.12). The Fermi-LAT highest energy for a photon associated with PKS 0736+017 (at the $95 \%$ confidence level) is $106 \mathrm{GeV}$ (detected on MJD 57072 at 14:42:20 UTC). This is also the highest photon energy when considering the longer two-month interval described above. Given the high significance of the detection, a Fermi-LAT

\footnotetext{
3 https://fermi.gsfc.nasa.gov/ssc/data/access/lat/msl_ lc/

4 See http://fermi.gsfc.nasa.gov/ssc/data/analysis/ documentation

5 The best-fit $\gamma$-ray spectrum of 3FGL J0739.4+0137 provided in the 3FGL catalog is a log-parabola with index $a=2.25 \pm 0.05$, curvature $b=$ $0.17 \pm 0.03$, reference energy $E_{0}=327.1 \mathrm{MeV}$, and $E F_{(1 \mathrm{GeV}-100 \mathrm{GeV})}=$ $(3.66 \pm 0.13) \times 10^{-11} \mathrm{erg} \mathrm{cm}^{-2} \mathrm{~s}^{-1}$. The Fermi-LAT Collaboration has recently released a new $\gamma$-ray catalog, the 4FGL (Fermi-LAT Collaboration 2019). The 4FGL associated source for PKS 0736+ 017, 4FGL J0739.2+0137, is again best described by a log-parabolic spectrum, with $a=2.33 \pm 0.02$, curvature $b=0.09 \pm 0.01$, reference energy $E_{0}=503.3 \mathrm{MeV}$, and $E F_{(100 \mathrm{MeV}-100 \mathrm{GeV})}=(6.12 \pm 0.14) \times$ $10^{-11} \mathrm{erg} \mathrm{cm}^{-2} \mathrm{~s}^{-1}$.
}

data analysis was also performed in a time window strictly simultaneous with the H.E.S.S. detection (MJD 57072, 18:5320:55 UTC). PKS $0736+017$ is detected in this dataset with a $\mathrm{TS}=37(\sim 6 \sigma)$, a flux of $F_{(100 \mathrm{MeV}-500 \mathrm{GeV})}=(4.57 \pm 1.52) \times$ $10^{-6} \mathrm{~cm}^{-2} \mathrm{~s}^{-1}$, and a photon index of $\Gamma=2.43 \pm 0.33$. The highest energy LAT photon simultaneous with the H.E.S.S. detection has $E=1.71 \mathrm{GeV}$ (detected on MJD 57072, at 20:40:16 UTC). Figure 1 shows the Fermi-LAT spectra of PKS 0736+017 for the two time windows described here.

To estimate the variability in the HE $\gamma$-ray band, the fractional variability $F_{\text {var,HE }}$ of the 12-h binned light curve was computed following Vaughan et al. (2003). This results in $F_{\mathrm{var}, \mathrm{HE}}=(62 \pm$ $7) \%$, clearly supporting the presence of variability of the HE $\gamma$ ray flux. This finding does not depend on the binning of the light curve: for a 6-h binned light curve, $F_{\text {var,HE }}=(48 \pm 2) \%$, while for a 24-h binned light curve, $F_{\mathrm{var}, \mathrm{HE}}=(57 \pm 4) \%$. An important property of a flare is its variability timescale, which is defined here as the flux-doubling timescale (see, e.g., Aliu et al. 2016):

$F(t)=\frac{a}{2^{-\left(t-t_{0}\right) / b}+2^{\left(t-t_{0}\right) / c}}$,

where $t_{0}$ is the time of the peak flux in the light curve, $a$ fits the peak flux, and $b$ and $c$ denote the rising and falling flux-doubling timescales, respectively. The evolution of the $\gamma$-ray flare in the HE band shows a slow flux increase starting on February 13, 2015, with a first flare on February 18, 2015, at an integral flux level of around $3 \times 10^{-6} \mathrm{~cm}^{-2} \mathrm{~s}^{-1}$. The flux then dropped by a factor of around four, before brightening again on February 19, 2015, coincidentally with the H.E.S.S. detection. The flux doubling timescale for this second flare centered on the H.E.S.S. detection is fitted using the 6-h binned light curve as $6 \pm 4 \mathrm{~h}$ for the rising and $4 \pm 2 \mathrm{~h}$ for the falling part of the flare.

\subsection{Swift-XRT}

Following the detection of the $\gamma$-ray flaring activity in PKS 0736+017, ToO observations were requested to the Neil Gehrels Swift satellite (Gehrels et al. 2005) in order to measure the flux of PKS $0736+017$ in soft X-rays and the optical-UV. The ToO resulted in four observations with Swift starting from February 20, 2015, for a total exposure time of around $12.3 \mathrm{ks}$. The details are provided in Table 2.

Data from Swift-XRT (Burrows et al. 2005) are analyzed using Heasoft version 6.18. All observations are taken in the standard Photon Counting observing mode. The event files are cleaned using standard screening criteria. Images, spectra, and light curves are extracted using a circular region with radius equal to 20 pixels. The background is extracted from an annular region with inner radius equal to 50 pixels, and outer radius equal to 160 pixels. The count rates are around $0.11-0.16 \mathrm{~s}^{-1}$, and are low enough that no pile up affects the analysis.

Spectral analyses of Swift-XRT data are performed using XSpec version 12.9 . The ancillary response files are recomputed using xrtmkarf, while the redistribution matrix files provided by the Swift team are used. Data are rebinned using grppha imposing a minimum of 30 counts per bin, and data below $0.3 \mathrm{keV}$ are excluded.

The X-ray spectrum from each of the four Swift-XRT observations is fitted with an absorbed power-law model (phabs*cflux*powerlaw within Xspec, to access the unabsorbed flux in the $0.3-10 \mathrm{keV}$ band) to take into account the absorption by neutral material in the Milky Way. The value of $N_{\mathrm{H}}$ in the direction of PKS $0736+017$ is fixed to $7.8 \times 10^{20} \mathrm{~cm}^{-2}$ as estimated by Dickey \& Lockman (1990). The results of the fit 
Table 2. Details of the Swift observations of PKS 0736+017.

\begin{tabular}{lcccccc}
\hline \hline Obs. ID & $\begin{array}{c}\text { Starting time } \\
{[\mathrm{UTC}]}\end{array}$ & $\begin{array}{c}\text { Exposure } \\
{[\mathrm{ks}]}\end{array}$ & $\begin{array}{c}\text { Power-law } \\
\text { index }\end{array}$ & $\begin{array}{c}\text { Flux } 0.3-10 \mathrm{keV} \\
{\left[10^{-12} \mathrm{erg} \mathrm{cm}^{-2} \mathrm{~s}^{-1}\right]}\end{array}$ & $\chi^{2} /$ d.o.f. & UVOT filters \\
\hline 00033535009 & Feb. 20, 2015, 19:26 & 3.4 & $1.62 \pm 0.08$ & $8.4 \pm 0.5$ & $11.2 / 14$ & $V, B, U, W 1, W 2, M 2$ \\
00033535010 & Feb. 22, 2015, 01:46 & 2.5 & $1.49 \pm 0.09$ & $9.2 \pm 0.6$ & $13.7 / 10$ & $V, B, U, W 1, W 2, M 2$ \\
00033535011 & Feb. 22, 2015, 21:25 & 2.6 & $1.46 \pm 0.13$ & $7.3 \pm 0.7$ & $5.4 / 6$ & $W 1$ \\
00033535012 & Feb. 27, 2015,08:01 & 3.8 & $1.34 \pm 0.09$ & $6.9 \pm 0.6$ & $9.4 / 11$ & $U$ \\
\hline
\end{tabular}

are provided in Table 2. The Swift-XRT light curve is shown in Fig. 2, panel d. No significant variability is detected in the X-ray data: the four Swift-XRT observations are consistent with a constant flux $\left(\chi^{2} /\right.$ d.o.f. $=9 / 3$, which corresponds to a chance probability of about $3 \%)$, with an average $F_{0.3-10 \mathrm{keV}}=(8.1 \pm$ 0.3) $10^{-12} \mathrm{erg} \mathrm{cm}^{-2} \mathrm{~s}^{-1}$.

\subsection{Swift-UVOT}

Simultaneously with Swift-XRT, the UVOT telescope (Roming et al. 2005) also observed PKS 0736+017, guaranteeing a coverage in the optical and UV bands. During the first two observations all UVOT filters were available, while the third and fourth observation was taken only with the $W 1$ and $U$ filter, respectively (see Table 2). Fluxes were calculated using uvotmaghist, integrating over a circular region $5^{\prime \prime}$ in radius for the source, and $20^{\prime \prime}$ in radius for the background. Data are de-reddened following Roming et al. (2009) using $E_{B-V}=0.121$ as the value for the Galactic extinction (Schlafly \& Finkbeiner 2011). The SwiftUVOT light curve is presented in Fig. 2, panel e. There is clear variability in the optical band, showing a decrease from February 20 to February 27, 2015.

\subsection{ATOM}

The ATOM telescope (Hauser et al. 2004), located at the H.E.S.S. site, regularly observes PKS $0736+017$ as part of its blazar monitoring program, providing a long-term light curve of the source in the $R$ band. Fluxes from ATOM observations were extracted using a 4 " radius aperture, and were de-reddened using the same value of Galactic extinction $E_{B-V}=0.121$ as for the UVOT data analysis. There are no ATOM observations from the night of February 19, 2015, when H.E.S.S. detected VHE emission from PKS 0736+017. On the other hand, ATOM observations on the night of February 20, 2015, are contemporaneous with the UVOT observations. The light curve from ATOM data is provided in Fig. 2, panel e.

\subsection{Steward Observatory}

PKS $0736+017$ is among the sources covered by the Steward Observatory blazar monitoring program (Smith et al. 2009; Carnerero et al. 2015). The program provides optical monitoring for bright Fermi-LAT blazars, including photometry, spectroscopy, and spectro-polarimetry. During February 2015, PKS 0736+017 was observed on nine occasions from February 12 to February 20, using the $1.5 \mathrm{~m}$ Kuiper Telescope located on Mt. Bigelow, Arizona and the SPOL CCD spectro-polarimeter (Schmidt et al. 1992). The SPOL apertures range was 5.1" $\times 10^{\prime \prime}$; the first number is the width of the slit and the second number is the width of the spectroscopic extraction aperture. The extraction aperture is in the east-west direction on the sky. For the SPOL differential photometry measurements, the slit width used was $12.7^{\prime \prime}$ and the extraction aperture was $10^{\prime \prime}, 11^{\prime \prime}$, or $12^{\prime \prime}$, depend-

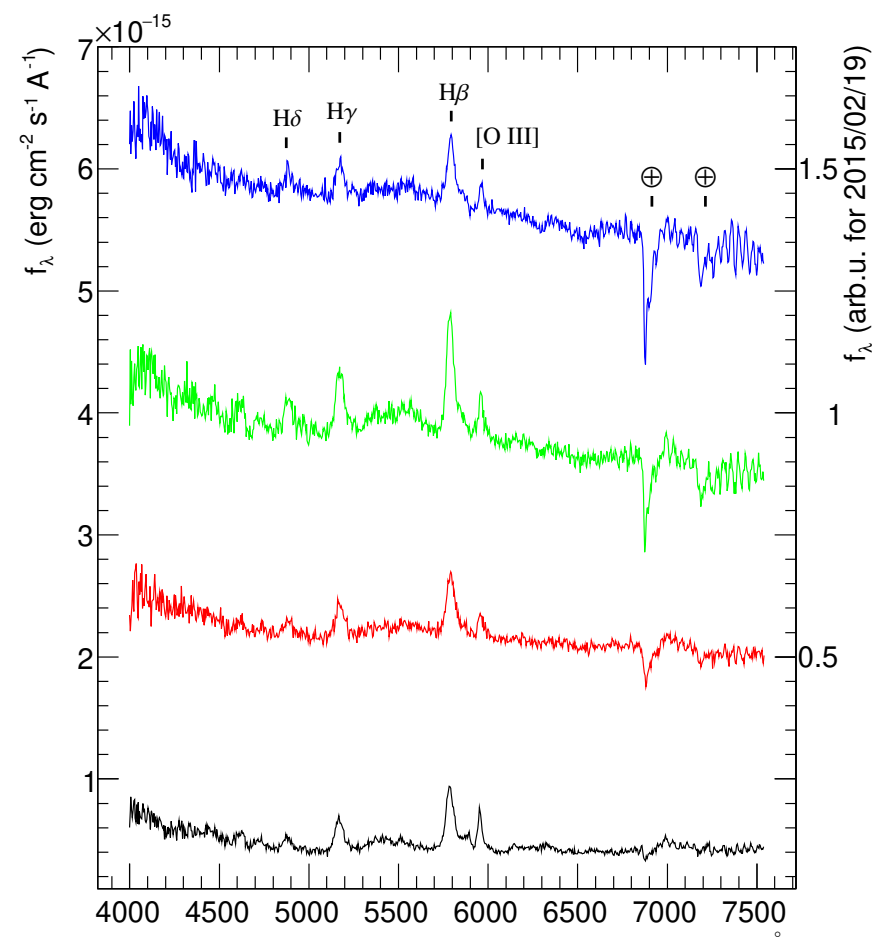

$\lambda(\AA)$

Fig. 3. Optical spectra (4000-7500 $\AA$ ) of PKS $0736+017$ obtained with SPOL on three consecutive nights in 2015: February 18 (in red), February 19 (in green), and February 20 (in blue). Due to the absence of photometric conditions on February 19, this spectrum is provided in arbitrary units. For reference, shown in black is a spectrum from a low state of the source (February 9, 2016). The emission lines and telluric features are identified in the spectrum from February 20, 2015.

ing on the seeing at the time of the measurement. Photometry measurements are provided in the $V$ (Johnson) and $R$ (KronCousins) bands, and were de-reddened using $E_{B-V}=0.121$ as for the UVOT and ATOM data. The light curve from the Steward Observatory monitoring of PKS $0736+017$ is shown in Fig. 2, and complements UVOT and ATOM measurements to provide optical coverage of the $\gamma$-ray flaring activity in PKS $0736+017$. Observations using SPOL were made on the morning of February 20, 2015 UTC, 8.4 h after the H.E.S.S. detection, and show a clear optical flare. There was an increase in luminosity of about one magnitude compared to the measurements taken $48 \mathrm{~h}$ previously with SPOL and with the Swift-UVOT and ATOM observations made $17 \mathrm{~h}$ afterwards. Measurements of the equivalent widths of the $\mathrm{H} \beta$ and $\mathrm{H} \gamma$ broad emission lines of PKS 0736+017 using the flux spectra obtained with SPOL (see Fig. 3) confirm the rapid brightening of the blazar on February 20. The decrease in the equivalent widths of the emission lines from February 19 to February 20 is consistent with a $1 \mathrm{mag}$ increase in the continuum brightness over the period of about $24 \mathrm{~h}$. The onset 
of the optical flare appears to be as rapid as its decay (see panel e in Fig. 2), and the coincidence of the optical, HE, and VHE outbursts is strong evidence that they are closely related physically.

Measurements of the optical polarization fraction and position angle are shown in panels $\mathrm{f}$ and $\mathrm{g}$ of Fig. 2. Uncertainties on both quantities are smaller than the symbols used: the uncertainty on the polarization fraction is $\leq 0.2 \%$, while the uncertainty on the angle is $\leq 1^{\circ}$. The polarization fraction reported was corrected by subtracting the assumed unpolarized contributions to the spectrum by the BLR and big blue bump. This is best done by subtracting an optical spectrum of PKS 0736+017 obtained during a faint period when the blazar shows little polarization. This is when the BLR and thermal optical continuum are most dominant in the spectrum. After subtracting the unpolarized flux, the measured polarization fraction is a better approximation to the intrinsic polarization of the synchrotron continuum responsible for the rapid optical flux variations. Given that the spectrum of PKS $0736+017$ still includes some polarization from the nonthermal continuum even when it is optically faint, the values of the polarization fraction plotted in Fig. 2 are the highest possible intrinsic polarization levels of the synchrotron emission. The highest polarization was not observed for the night of the optical flare, but for the night before at $(12.5 \pm 0.1) \%$. The polarization position angle was extremely variable during the week leading up to the $\gamma$-ray flare and was observed throughout the full $180^{\circ}$ range. It is interesting to note that the two measurements taken on February 20 show a variation of the polarization angle of $(44.9 \pm 0.6)^{\circ}$ in three hours, which is a very rapid rotation even for a blazar (see Blinov et al. 2015, 2016; Lyutikov \& Kravchenko 2017).

Spectro-polarimetry of PKS 0736+017 yields the spectral index $\alpha$ (defined as $f_{\lambda} \propto \lambda^{-\alpha}$ ) of the optical synchrotron continuum during the flare, which is key to constraining radiative models (see Sect. 3.2). The spectral index is derived directly from the polarized flux spectrum, assuming that the polarization of the synchrotron continuum is constant with wavelength. This may not be the case, but observations of BL Lac objects, where the synchrotron emission dominates the optical flux, do not often show strong wavelength-dependent polarization (Sitko et al. 1985; Jannuzi et al. 1994; Smith 1996). In addition, PKS 0736+017 shows no evidence of a strong variation in polarization position angle with wavelength in 2015 February. The de-reddened polarized flux spectrum of PKS 0736+017 is consistent with a featureless power law and indicates that the emission lines are unpolarized. During the morning of February 20, 2015 , the spectral index was found to be $1.0 \pm 0.1$, significantly flatter than for the nights before the flare, when it was observed to be between 1.4 and 1.8 , indicating a shift of the synchrotron peak frequency to higher energies.

\subsection{ASAS-SN and KAIT}

PKS $0736+017$ is also regularly monitored in the optical by the ASAS-SN project (Shappee et al. 2014) and the KAIT telescope (Cohen et al. 2014). Their public data were retrieved for the observations around the February $2015 \gamma$-ray flare. ASAS-SN $V$-magnitudes, and KAIT unfiltered magnitudes (which are close to the $R$ values; see $\mathrm{Li}$ et al. 2003) were converted to fluxes and de-reddened using $E_{B-V}=0.121$ as for the other optical observations. ASAS-SN and KAIT observed PKS 0736+017 during the morning (UTC) of February 19, respectively $11.5 \mathrm{~h}$ and $14 \mathrm{~h}$ before the H.E.S.S. VHE detection, and complement the optical light curve from UVOT, ATOM, and Steward Observatory.
The contribution of the host-galaxy to the optical measurements has not been investigated. The emission in the optical band is dominated by the non-thermal continuum from the quasar, as is evident from the SED shown in Fig. 5.

\section{Discussion}

\subsection{Location of the $\gamma$-ray emission region}

As shown in the multi-wavelength light curve in Fig. 2, the only instrument which was observing PKS 0736+017 together with H.E.S.S. during the night of February 19, 2015, was Fermi-LAT. Observations by Swift covered only the post-flare period, and there are no optical observations available within 8 hours of the H.E.S.S. detection. It is thus not possible to follow the usual approach of modeling the simultaneous SED to access blazar physics. In particular, there is no coverage of the behavior of the synchrotron component of PKS 0736+017 simultaneously with the $\gamma$-ray flare. On the other hand, constraints can be put on the location of the emitting region $r$ (defined as the distance from the $\mathrm{SMBH}$ ) within the relativistic jet, under some hypotheses. Here the following is assumed: ${ }^{6}$

- The main radiation mechanism responsible for the $\gamma$-ray emission is inverse-Compton scattering by a population of electrons and positrons in the jet, off a low-energy photon field represented by the radiation from the accretion disk, the BLR, and the dusty torus.

- The $\gamma$-ray emission during the flare is produced from a single region within the relativistic jet.

- The jet of PKS $0736+017$ is closely aligned to the line of sight, and the Doppler factor $\delta$ of the emitting region equals its bulk Lorentz factor $\Gamma$ (i.e., the angle to the line of sight $\vartheta_{\text {LOS }}$ is equal to $1 / \Gamma)$.

- The emitting region is approximated by a spherical plasmoid in the jet, characterized by its radius $R^{\prime}$, which is related to the variability timescale $\tau_{\text {var }}$ via $R^{\prime} \simeq c \tau_{\text {var }} \frac{\Gamma}{1+z}$, where $c$ is the speed of light in vacuum and $z$ is the redshift of the source. The most constraining estimate for $\tau_{\text {var }}$ comes from the falling part of the Fermi-LAT flare as $\tau_{\text {var }}=(4 \pm 2) \mathrm{h}$; the two extreme values of 2 and $6 \mathrm{~h}$ are used in the following.

- The emitting region fills the entire cross section of the relativistic jet, and thus the location of the emitting region $r$ and its size $R$ are simply related by $R / r=\tan \vartheta_{\text {open }}$, where $\vartheta_{\text {open }}$ is the jet opening angle.

- The location of the BLR $r_{\mathrm{BLR}}$ is derived from the luminosity of the $\mathrm{H} \beta$ line, which is measured from Steward Observatory data to be $L_{\mathrm{H} \beta}=4.20 \times 10^{42} \mathrm{erg} \mathrm{s}^{-1}$. Following Greene \& Ho (2005), $r_{\text {BLR }}$ is then estimated as $1.45 \times 10^{17} \mathrm{~cm}$. The total BLR luminosity is similarly estimated from $L_{\mathrm{H} \beta}$ (see Finke 2016) as $L_{\mathrm{BLR}}=1.24 \times 10^{44} \mathrm{erg} \mathrm{s}^{-1}$, and $L_{\mathrm{disk}} \simeq 10 L_{\mathrm{BLR}}=1.24 \times$ $10^{45} \mathrm{erg} \mathrm{s}^{-1}$. The location of the dusty torus $r_{\text {torus }}$ is assumed to scale as $r_{\text {torus }} \simeq 2.5 \times 10^{18} \sqrt{L_{\text {disk }} / 10^{45} \mathrm{erg} \mathrm{s}^{-1}}=2.85 \times 10^{18} \mathrm{~cm}$ (see, e.g., Sikora et al. 2009; Hayashida et al. 2012). The values for the luminosity of the accretion disk and the SMBH mass of PKS 0736+017 vary largely in the literature: estimates for $L_{\text {disk }}$ range from $10^{44.6}$ to $10^{45.7} \mathrm{erg} \mathrm{s}^{-1}$, while estimates for $M_{\bullet}$ range from $10^{8}$ to $10^{8.7} M_{\odot}$ (Wandel 1991; McLure \& Dunlop 2001; Woo \& Urry 2002; Marchesini et al. 2004; Dai et al. 2007). The adopted value of $L_{\text {disk }} \simeq 1.24 \times 10^{45} \mathrm{erg} \mathrm{s}^{-1}$ is consistent with these previous estimates.

\footnotetext{
6 Here and in the following, quantities in the co-moving jet frame are indicated by a prime.
} 
- The BLR is modeled as a spherical shell centered at $r_{\mathrm{BLR}}$, with lower boundary $r_{\text {in }}=0.9 * r_{\mathrm{BLR}}$ and outer boundary $r_{\mathrm{out}}=$ $1.1 * r_{\mathrm{BLR}}$. As discussed in Böttcher \& Els (2016), the choice of the boundaries have negligible effects on the BLR opacity. The opening angle of the dusty torus is assumed to be $\pi / 4$ as in Nalewajko et al. (2014).

The first constraint on the location of the $\gamma$-ray emitting region comes from opacity to $\gamma-\gamma$ pair-production. The VHE photons can pair-produce over the bright environment of the SMBH and, as shown by several authors (see, e.g., Donea \& Protheroe 2003; Liu \& Bai 2006; Reimer 2007; Tavecchio \& Ghisellini 2012; Böttcher \& Els 2016; Finke 2016), the absorption can be so severe that the simple detection of VHE photons can be used to exclude an emitting region located at the base of the jet. The $\gamma$-ray spectra presented in Fig. 1 show that the extrapolated Fermi-LAT spectrum, once the absorption on the EBL is taken into account, is consistent with the H.E.S.S. detection. This translates into an internal opacity equal to zero, constraining the location of the emitting region well beyond $r_{\mathrm{BLR}}$. However, given the large uncertainty in the simultaneous Fermi-LAT spectrum, the most conservative opacity constraint has to be computed using the upper end of the Fermi-LAT extrapolated bowtie. In this case a break exists between the Fermi-LAT and H.E.S.S. energy band. This break can be intrinsic (for example due to a break in the lepton population, or due to the transition from the Thomson to the KleinNishina regime of the inverse-Compton scattering), or due to additional pair-production absorption at the source. In particular, while pair-production on the infrared photons from the dusty torus is expected to produce a spectral break at a few $\mathrm{TeV}$, absorption on Ly $\alpha$ photons can produce a spectral break at around $100 \mathrm{GeV}$. Given that it is not possible to discriminate between an intrinsic cutoff in the $\gamma$-ray emission and an absorption effect, the opacity argument can only be used to put a lower limit on the location of the emitting region $r_{\min }$ : if the emitting region is located farther in closer to the SMBH, the spectral break between the Fermi-LAT and H.E.S.S. observations would have been stronger. To quantitatively estimate $r_{\min }$, the model described in Böttcher \& Els (2016) is used, together with the values of $L_{\text {disk }}$ and $r_{\mathrm{BLR}}$ provided above. The calculation of $r_{\min }$ is performed by extrapolating into the VHE band the upper end of the Fermi-LAT bowtie, including absorption by internal and EBL photons, and varying $r$ until the extrapolated spectrum matches the H.E.S.S. measurement. This estimate results in $r_{\text {min }}=1.6 \times 10^{17} \mathrm{~cm}$, or $1.1 r_{\mathrm{BLR}}$. This limit is shown in Fig. 4 by the red exclusion region.

The second constraint comes from the collimation of the relativistic jet. As shown by radio observations, relativistic jets from SMBHs are highly collimated, and in particular $\Gamma \vartheta_{\text {open }}<1$. In the following the reformulation by Nalewajko et al. (2014) is used, which translates this inequality into a limit in the $\Gamma-r$ plane, under the assumption that $R^{\prime} \simeq c \tau_{\operatorname{var}} \frac{\Gamma}{1+z}$, and that $R^{\prime} / r=\tan \vartheta_{\text {open }}$. This constraint is shown in Fig. 4 by the blue exclusion region.

The last constraint comes from the cooling of the leptons due to inverse-Compton scattering. In particular, the cooling timescale $\tau_{\text {cool }}$ is required to be shorter than the observed variability timescale $\tau_{\text {var }}$, under the assumption again that $R^{\prime} \simeq$ $c \tau_{\text {var }} \frac{\Gamma}{1+z}$, and that the cooling timescale is dominated by the inverse-Compton scattering on the external photon fields. To translate this condition into an exclusion region in the $\Gamma-r$ plane, we used a modified version of the equations described in Nalewajko et al. (2014); instead of considering a BLR opening angle of $\pi / 4$, as assumed in the original paper, the equations were recomputed for a spherical BLR to be consistent with the opacity constraint. The impact of this change is to open up the parameter space, allowing a larger range of $\Gamma$ values when the emitting region

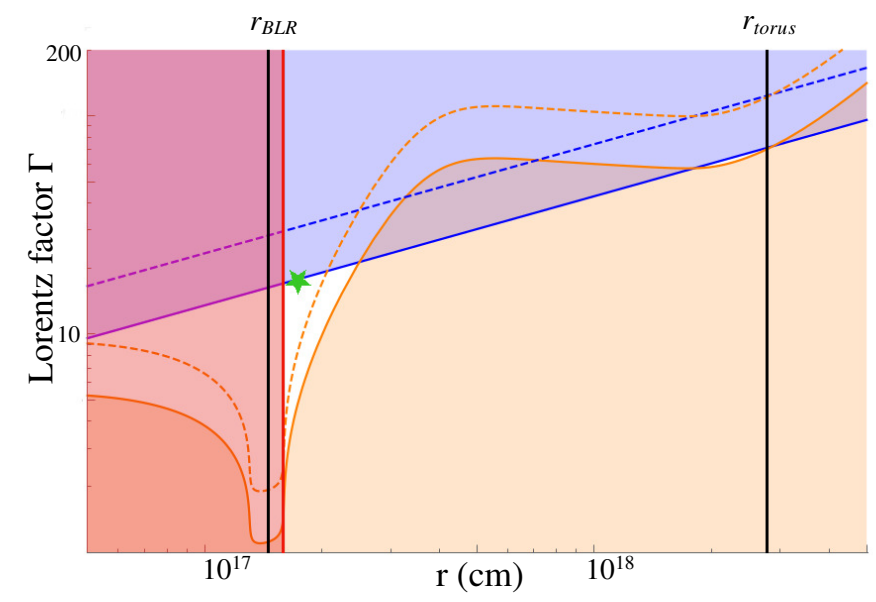

Fig. 4. Constraint on the location of the emitting region $r$ in centimeters, defined as the distance from the $\mathrm{SMBH}$, as a function of the bulk Lorentz factor $\Gamma$. The red exclusion region represents the opacity constraint, the blue exclusion region represents the collimation constraint, and the orange exclusion region represents the cooling constraint, the last two computed for $\tau_{\mathrm{var}}=6 \mathrm{~h}$. Dashed lines show how the constraints change assuming $\tau_{\mathrm{var}}=2 \mathrm{~h}$. The white regions indicate the allowed part of the parameter space. The vertical black lines represents the estimated locations of the BLR $r_{\mathrm{BLR}}$ and of the dust torus $r_{\text {torus }}$. The green star identifies the parameters of the EIC solution discussed in Sect. 3.2 and plotted in Fig. 5.

is located close to $r_{\mathrm{BLR}}$. The cooling constraint is shown in Fig. 4 by the orange exclusion region. The cooling timescale depends on the energy density of the target photons, and thus depends on $r$ : the shape of the exclusion region in Fig. 4 is due to the changes in the photon field seen by the $\gamma$-ray emitting region when approaching the BLR radius $r_{\mathrm{BLR}}$ and torus radius $r_{\text {torus }}$.

These three constraints significantly limit the $\Gamma-r$ plane. A location of the emitting region close to the $\mathrm{SMBH}$, where the dominant photon field is the thermal radiation from the accretion disk, is excluded due to the opacity constraint. However, two scenarios are allowed: an emitting region located at around $r_{\mathrm{BLR}}$, with $\Gamma \simeq 10-20$, and an emitting region close to $r_{\text {torus }}$, with $\Gamma \simeq$ 60. In the first scenario, the dominant external photon field is the emission from the BLR, while in the second case it is the thermal emission from the dusty torus. The first solution implies lower values of $\Gamma$, in line with radio observations of $\gamma$-ray FSRQs and of PKS 0736+017 in particular, for which an estimate of $\Gamma=$ 16.5-17.0 is provided by Pushkarev et al. (2009) and Hovatta et al. (2009), respectively, from direct measurements of the jet speed. Changing the assumed value of $\tau_{\text {var }}$ results in a translation of the constraints towards higher values of $\Gamma$, as shown in Fig. 4.

\subsection{Spectral energy distribution}

The SED of PKS 0736+017 during the February 2015 flaring activity, using the data described in the previous section and together with archival observations ${ }^{7}$, is presented in Fig. 5. As discussed previously, it is not possible to fit the overall SED during the night of the H.E.S.S. detection due to the absence of simultaneous information on the behavior of the synchrotron component. It is, however, interesting to use the constraints on $\Gamma$ and $r$ computed in the previous section as input parameters for a leptonic model fitting the $\gamma$-ray SED. The goal is first to see if it is possible to find a model that remains consistent with the observations during the flare, and for what parameter

\footnotetext{
From the SSDC SED builder, https://tools. ssdc. asi.it
} 


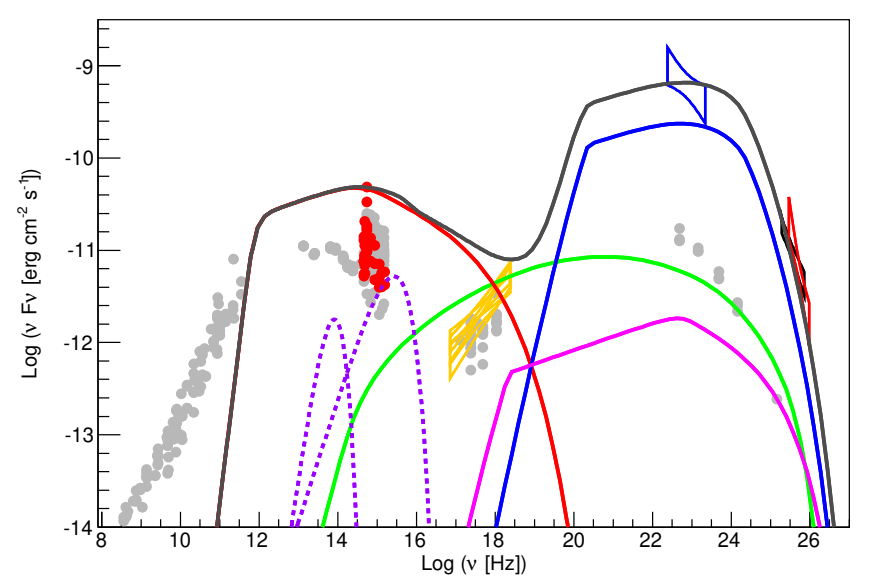

Fig. 5. Spectral energy distribution of PKS 0736+017 during the multiwavelength campaign of February 2015, together with archival data (in gray). The black and red bowties represent the H.E.S.S. spectra during the night of February 19, 2015, for the monoscopic and stereoscopic reconstruction, respectively; the blue bowtie represents the Fermi-LAT spectrum simultaneous with the H.E.S.S. detection; the orange bowties represents the four Swift-XRT spectra, acquired after the H.E.S.S. detection; the red points represent all optical-UV measurements before and after the flare, from ATOM, Steward Observatory, and Swift-UVOT. Shown are the EIC model components, from low to high energies: synchrotron emission by leptons (in red), synchrotron self-Compton emission (in green), EIC emission over Ly $\alpha$ line (in blue, emission from EIC over other BLR lines is not plotted for the sake of clarity), EIC emission over the dust torus (in magenta). The violet dotted lines represent the thermal emission from the dusty torus and the accretion disk.

values (other than $\Gamma$ and $r$ ), and then discuss the implications for the behavior of the synchrotron component during the flare. For this purpose, the $\gamma$-ray emitting region is assumed to be at $r=1.7 \times 10^{17} \mathrm{~cm}$, and located at the limit of the collimation constraint (i.e., $\Gamma \vartheta_{\text {open }}=1$; the solution is identified as a green star in Fig. 4). The Lorentz factor $\Gamma$ is equal to 17.7, and the size of the emitting region $R$ is estimated via $\tau_{\text {var }}=6 \mathrm{~h}$ to be $9.6 \times 10^{15} \mathrm{~cm}$. The ratio $R / r$ gives a jet opening angle $\vartheta_{\text {open }}$ of $3.2^{\circ}$ (which by construction respects $\Gamma \vartheta_{\text {open }}=1$ ). The estimate of the jet opening angle of PKS $0736+017$ from radio observations is smaller, $\vartheta_{\text {open }}=1.8^{\circ} \pm 0.3^{\circ}$ (Pushkarev et al. 2017). This value refers, however, to the jet opening angle as measured at much larger $(\mathrm{kpc})$ distances from the SMBH. The fact that the intrinsic opening angle gets smaller downstream is consistent with the findings of Pushkarev et al. (2017) from an analysis of 65 AGNs. The leptons in the emitting region scatter primarily BLR photons: the BLR photon energy density at $r$ is estimated following Nalewajko et al. (2014) resulting in $u_{\mathrm{BLR}}=3.95 \mathrm{erg} \mathrm{cm}^{-3}$. The energy density of torus photons is similarly estimated as $u_{\text {torus }}=0.005 \mathrm{erg} \mathrm{cm}^{-3}$, clearly negligible with respect to the BLR value. The numerical code used to compute synchrotron and EIC radiation is described in Cerruti et al. (2013). The electron distribution in the emitting region is modeled by a broken power-law function with exponential cut-off

$N_{\mathrm{e}}^{\prime}\left(\gamma^{\prime}\right)= \begin{cases}N_{0}^{\prime} \gamma^{\prime-n_{1}} & \text { if } \gamma_{\text {min }}^{\prime} \leq \gamma^{\prime} \leq \gamma_{\text {break }}^{\prime} \\ N_{0}^{\prime} \gamma_{\text {break }}^{\prime\left(n_{2}-n_{1}\right)} \gamma^{\prime-n_{2}} \mathrm{e}^{-\gamma^{\prime} / \gamma_{\text {Max }}^{\prime}} & \text { if } \gamma_{\text {break }}^{\prime}<\gamma^{\prime}\end{cases}$

with indices $n_{1}$ and $n_{2}$ below and above the break Lorentz factor $\gamma_{\text {break }}^{\prime}$, cutoff Lorentz factor $\gamma_{\text {Max }}^{\prime}$, and minimum Lorentz factor $\gamma_{\text {min }}^{\prime}=10$. A complex BLR line spectrum is assumed, using the seven most prominent emission lines from the average quasar model by Telfer et al. (2002). The absorption on BLR photons is calculated following Dermer et al. (2009), under the assumption that the most relevant absorption is the one due to the Ly $\alpha$ line. Absorption by the EBL is computed using the model by Franceschini et al. (2008). The remaining free parameters are adjusted to reproduce the $\gamma$-ray data from Fermi-LAT and H.E.S.S., a peak of the synchrotron component $v_{\text {sync }}=8 \times$ $10^{14} \mathrm{~Hz}$, as constrained by the flat optical spectrum measured by Steward Observatory observations $8 \mathrm{~h}$ after the H.E.S.S. detection, and a flux of the synchrotron peak similar to the maximum observed optical flux. A good description of the SED can be obtained assuming $n_{1}=2.7, n_{2}=3.4, \gamma_{\text {break }}=2 \times 10^{3}$, $\gamma_{\text {Max }}=1 \times 10^{5}, N_{0}^{\prime}=1 \times 10^{6} \mathrm{~cm}^{-3}$, and a magnetic field $B^{\prime}=1.6 \mathrm{G}$. The corresponding value of the equipartition factor (the ratio of the electron energy density to the magnetic energy density) $u_{\mathrm{e}}^{\prime} / u_{B}^{\prime}$ is 2.3 . The resulting modeling is shown in Fig. 5. The peak of the EIC component of the model is in the $\mathrm{GeV}$ range, resulting in a flat spectrum in the LAT energy band. The hard HE spectrum is needed in order to fit the VHE spectrum, as both the transition to the Klein-Nishina regime and the absorption on BLR photons lead to an under-prediction of the VHE spectrum for softer HE spectra. The model indicates that an X-ray flare could have occurred simultaneously with the VHE $\gamma$-ray flare, with significant softening of the spectrum, due to the emergence of the synchrotron component, similar to the one observed in PKS 1441+25 (Abeysekara et al. 2015; Ahnen et al. 2015). Although the model predicts a soft X-ray flare a factor of about ten brighter at $0.1 \mathrm{keV}$ compared to the Swift-XRT observations of PKS $0736+017$, it is important to note that the electron synchrotron cooling timescale at these energies is very short. For electrons with Lorentz factor $\gamma=10^{4}$ in a magnetic field $B^{\prime}=1.6 \mathrm{G}$, and moving towards the observer with bulk Lorentz factor $\Gamma=17.7$, the synchrotron cooling timescale is only $35 \mathrm{~min}$, to be compared with the $24 \mathrm{~h}$ delay of the SwiftXRT observations with respect to the time of the H.E.S.S. detection. This prediction of an unseen soft-X-ray flare is solid with respect to the free parameters considered: having fixed $\Gamma, R$, and $u_{\mathrm{BLR}}$, the normalization of the particle distribution $N_{0}^{\prime}$ and the magnetic field $B^{\prime}$ are adjusted to reproduce the peak flux of the synchrotron and EIC components, and $\gamma_{\text {break }}^{\prime}$ is adjusted to have a synchrotron peak in the optical band. The high value of $\gamma_{\text {Max }}^{\prime}$, which is the parameter that implies the occurrence of a simultaneous X-ray flare from the source, is required for the model to reach VHE $\gamma$ rays.

\section{Conclusions}

H.E.S.S. observations of the FSRQ PKS 0736+017, triggered on the basis of a $\gamma$-ray flare detected with Fermi-LAT, resulted in the discovery of VHE emission from this quasar during the night of February 19, 2015. PKS $0736+017$ is the seventh member of the elusive population of FSRQs known to emit VHE photons, the nearest detected to date. Fermi-LAT and H.E.S.S. high-energy flares were accompanied by at least a 1 mag brightening of the non-thermal optical continuum. Fermi-LAT observations show the presence of a relatively fast $\gamma$-ray flare, with a flux-doubling timescale of around six hours. No optical or X-ray observations were performed strictly simultaneously with the H.E.S.S. detection. Nonetheless, both temporal and spectral measurements in the $\gamma$-ray band can be used to put model-dependent constraints on the location of the $\gamma$-ray emitting region in the jet, and on its Lorentz factor. The Fermi-LAT and the H.E.S.S. spectra collectively constrain the location of the emitting region to be located beyond $r=1.1 r_{\mathrm{BLR}}=1.6 \times 10^{17} \mathrm{~cm}$ to avoid absorption due to $\gamma-\gamma$ pair-production with BLR photons. A location of the 
$\gamma$-ray emitting region just outside the BLR with a Lorentz factor $\Gamma \simeq 10-20$ is thus a viable solution which satisfies both temporal and spectral constraints. Alternatively, the emitting region may be located much farther away, at around $r_{\text {torus }}=2.85 \times 10^{18} \mathrm{~cm}$, with a higher value of $\Gamma \simeq 60$, with electrons in the jet in this case scattering thermal photons from the dusty torus.

The main limitation of this study is the absence of strictly simultaneous observations of the behavior of the synchrotron component during the H.E.S.S. detection. With simultaneous optical, UV, and X-ray data, it will be possible to uniquely constrain the electron energy distribution, and the opacity and variability constraints could be coupled with a full SED fitting. In addition, with tighter constraints on the location of the $\gamma$-ray emitting region it will be possible to study in greater detail the opacity in the VHE band, potentially putting constraints on the geometry of the BLR itself (its aperture angle and its profile). This kind of study is more easily done using nearby quasars, for which the internal absorption is not hidden by the EBL absorption. In this context, PKS 0736+017 represents the ideal FSRQ, being located much closer than all other VHE FSRQs. Its location near the celestial equator also makes it a perfect target for all current ground-based $\gamma$-ray telescopes, with only marginal zenith-angle effects. The next $\gamma$-ray flare from PKS 0736+017 has thus the potential to be an extremely interesting event for the study of $\gamma$-ray quasars, and future multi-wavelength coordinated observing campaigns are strongly encouraged.

Acknowledgements. The support of the Namibian authorities and of the University of Namibia in facilitating the construction and operation of H.E.S.S. is gratefully acknowledged, as is the support by the German Ministry for Education and Research (BMBF), the Max Planck Society, the German Research Foundation (DFG), the Helmholtz Association, the Alexander von Humbold Foundation, the French Ministry of Higher Education, Research and Innovation, the Centre National de la Recherche Scientifique (CNRS/IN2P3 and CNRS/INSU), the Commissariat à l'énergie atomique et aux énergies alternatives (CEA), the UK Science and Technology Facilities Council (STFC), the Knut and Alice Wallenberg Foundation, the National Science Centre, Poland grant no. 2016/22/M/ST9/00382, the South African Department of Science and Technology and National Research Foundation, the University of Namibia the National Commission on Research, Science and Technology of Namibia (NCRST), the Austrian Federal Ministry of Education, Science and Research and the Austrian Science Fund (FWF), the Australian Research Council (ARC), the Japan Society for the Promotion of Science and by the University of Amsterdam. We appreciate the excellent work of the technical support staff in Berlin, Zeuthen Heidelberg, Palaiseau, Paris, Saclay, Tübingen and in Namibia in the construction and operation of the equipment. This work benefited from services provided by the H.E.S.S. Virtual Organisation, supported by the national resource providers of the EGI Federation. The CC-IN2P3 (cc.in2p3.fr) is gratefully acknowledged for providing a significant amount of the computing resources and services needed for this work. Part of this work is based on archival data software or online services provided by the Space Science Data Center - ASI Blazar observations at Steward Observatory are funded though NASA/Ferm Guest Investigator Program grant NNX15AU81G. Matteo Cerruti has received financial support through the Postdoctoral Junior Leader Fellowship Programme from la Caixa Banking Foundation (LCF/BQ/PI18/11630012). The authors would like to thank the anonymous referee, whose constructive comments significantly improved the manuscript.

\section{References}

Abdo, A. A., Ackermann, M., Agudo, I., et al. 2010, ApJ, 716, 30 Abeysekara, A. U., Archambault, S., Archer, A., et al. 2015, ApJ, 815, L22 Acero, F., Ackermann, M., Ajello, M., et al. 2015, ApJS, 218, 23 Acero, F., Ackermann, M., Ajello, M., et al. 2016, ApJS, 223, 26 Agudo, I., Jorstad, S. G., Marscher, A. P., et al. 2011a, ApJ, 726, L13 Agudo, I., Marscher, A. P., Jorstad, S. G., et al. 2011b, ApJ, 735, L10 Aharonian, F., Akhperjanian, A. G., Barres de Almeida, U., et al. 2007, A\&A 475, L9

Ahnen, M. L., Ansoldi, S., Antonelli, L. A., et al. 2015, ApJ, 815, L23 Ahnen, M. L., Ansoldi, S., Antonelli, L. A., et al. 2016, A\&A, 595, A98 Aleksić, J., Antonelli, L. A., Antoranz, P., et al. 2011, ApJ, 730, L8
Aleksić, J., Ansoldi, S., Antonelli, L. A., et al. 2014, A\&A, 569, A46 Aliu, E., Archambault, S., Arlen, T., et al. 2014, ApJ, 782, 13 Aliu, E., Archambault, S., Archer, A., et al. 2016, A\&A, 594, A76 Atwood, W. B., Abdo, A. A., Ackermann, M., et al. 2009, ApJ, 697, 1071 Baldwin, J. A. 1975, ApJ, 201, 26

Becker, P. A., \& Kafatos, M. 1995, ApJ, 453, 83 Berge, D., Funk, S., \& Hinton, J. 2007, A\&A, 466, 1219

Blandford, R. D., \& Levinson, A. 1995, ApJ, 441, 79 Blandford, R. D., \& Rees, M. J. 1978, Phys. Scr., 17, 265 Błażejowski, M., Sikora, M., Moderski, R., \& Madejski, G. M. 2000, ApJ, 545, 107

Blinov, D., Pavlidou, V., Papadakis, I., et al. 2015, MNRAS, 453, 1669 Blinov, D., Pavlidou, V., Papadakis, I. E., et al. 2016, MNRAS, 457, 2252 Böttcher, M., \& Els, P. 2016, ApJ, 821, 102

Böttcher, M., Reimer, A., Sweeney, K., \& Prakash, A. 2013, ApJ, 768, 54 Brown, A. M. 2013, MNRAS, 431, 824

Burrows, D. N., Hill, J. E., Nousek, J. A., et al. 2005, Space Sci. Rev., 120, 165 Cao, G., \& Wang, J.-C. 2013, MNRAS, 436, 2170

Carnerero, M. I., Raiteri, C. M., Villata, M., et al. 2015, MNRAS, 450, 2677 Cerruti, M. 2015, ArXiv e-prints [arXiv:1501.03554]

Cerruti, M., Dermer, C. D., Lott, B., Boisson, C., \& Zech, A. 2013, ApJ, 771, L4 Clements, S. D., Jenks, A., \& Torres, Y. 2003, AJ, 126, 37

Cohen, D. P., Romani, R. W., Filippenko, A. V., et al. 2014, ApJ, 797, 137 Coogan, R. T., Brown, A. M., \& Chadwick, P. M. 2016, MNRAS, 458, 354 Dai, H., Xie, G. Z., Zhou, S. B., et al. 2007, AJ, 133, 2187 D’Ammando, F., \& Orienti, M. 2014, ATel, 6731

Day, G. A., Shimmins, A. J., Ekers, R. D., \& Cole, D. J. 1966, Aust. J. Phys., 19, 35

De Naurois, M., \& Rolland, L. 2009, Astropart. Phys., 32, 231

Dermer, C. D., \& Schlickeiser, R. 1993, ApJ, 416, 458

Dermer, C. D., \& Schlickeiser, R. 1994, ApJS, 90, 945

Dermer, C. D., Schlickeiser, R., \& Mastichiadis, A. 1992, A\&A, 256, L27

Dermer, C. D., Finke, J. D., Krug, H., \& Böttcher, M. 2009, ApJ, 692, 32

Dermer, C. D., Cerruti, M., Lott, B., Boisson, C., \& Zech, A. 2014, ApJ, 782, 82 Dickey, J. M., \& Lockman, F. J. 1990, ARA\&A, 28, 215

Donea, A.-C., \& Protheroe, R. J. 2003, Astropart. Phys., 18, 377

Dotson, A., Georganopoulos, M., Meyer, E. T., \& McCann, K. 2015, ApJ, 809, 164

Fermi-LAT Collaboration 2019, ApJS, submitted [arXiv:1902 . 10045]

Finke, J. D. 2016, ApJ, 830, 94

Finke, J. D., \& Dermer, C. D. 2010, ApJ, 714, L303

Franceschini, A., Rodighiero, G., \& Vaccari, M. 2008, A\&A, 487, 837

Gehrels, N., Chincarini, G., Giommi, P., et al. 2005, ApJ, 621, 558

Ghisellini, G., \& Tavecchio, F. 2009, MNRAS, 397, 985

Ghisellini, G., Tavecchio, F., Foschini, L., et al. 2010, MNRAS, 402, 497

Greene, J. E., \& Ho, L. C. 2005, ApJ, 630, 122

Hauser, M., Möllenhoff, C., Pühlhofer, G., et al. 2004, Astron. Nachr., 325, 659 Hayashida, M., Madejski, G. M., Nalewajko, K., et al. 2012, ApJ, 754, 114

H.E.S.S. Collaboration (Abramowski, A., et al.) 2013, A\&A, 554, A107

H.E.S.S. Collaboration (Abdalla, H., et al.) 2017, A\&A, 600, A89

H.E.S.S. Collaboration (Abdalla, H., et al.) 2019, A\&A, 627, A159

Ho, L. C., \& Kim, M. 2009, ApJS, 184, 398

Holler, M., Balzer, A., Chalmé-Calvet, R., et al. 2015, Int. Cosmic Ray Conf., 34,980

Hovatta, T., Valtaoja, E., Tornikoski, M., \& Lähteenmäki, A. 2009, A\&A, 494, 527

Jannuzi, B. T., Smith, P. S., \& Elston, R. 1994, ApJ, 428, 130

Jorstad, S. G., Marscher, A. P., Mattox, J. R., et al. 2001, ApJ, 556, 738

Kotilainen, J. K., Falomo, R., \& Scarpa, R. 1998, A\&A, 332, 503

Lanyi, G. E., Boboltz, D. A., Charlot, P., et al. 2010, AJ, 139, 1695

Lenain, J.-P. 2018, Astron. Comput., 22, 9

Li, T.-P., \& Ma, Y.-Q. 1983, ApJ, 272, 317

Li, W., Filippenko, A. V., Chornock, R., \& Jha, S. 2003, PASP, 115, 844

Lister, M. L., \& Homan, D. C. 2005, AJ, 130, 1389

Lister, M. L., Aller, H. D., Aller, M. F., et al. 2009, AJ, 137, 3718

Liu, H. T., \& Bai, J. M. 2006, ApJ, 653, 1089

Lyutikov, M., \& Kravchenko, E. V. 2017, MNRAS, 467, 3876

MAGIC Collaboration (Albert, J., et al.) 2008, Science, 320, 1752

MAGIC Collaboration (Acciari, V. A., et al.) 2018, A\&A, 619, A159

Malkan, M. A., \& Moore, R. L. 1986, ApJ, 300, 216

Marchesini, D., Celotti, A., \& Ferrarese, L. 2004, MNRAS, 351, 733

Marcowith, A., Henri, G., \& Pelletier, G. 1995, MNRAS, 277, 681

Mattox, J. R., Bertsch, D. L., Chiang, J., et al. 1996, ApJ, 461, 396

Max-Moerbeck, W., Hovatta, T., Richards, J. L., et al. 2014, MNRAS, 445, 428

McLure, R. J., \& Dunlop, J. S. 2001, MNRAS, 327, 199

McLure, R. J., Kukula, M. J., Dunlop, J. S., et al. 1999, MNRAS, 308, 377

Meyer, E. T., Fossati, G., Georganopoulos, M., \& Lister, M. L. 2012, ApJ, 752, L4 
Mirzoyan, R. 2017, ATel, 11061

Mukherjee, R., \& VERITAS Collaboration 2017, ATel , 11075

Nalewajko, K., Begelman, M. C., \& Sikora, M. 2014, ApJ, 789, 161

Padovani, P. 1992, MNRAS, 257, 404

Padovani, P., \& Giommi, P. 1995, ApJ, 444, 567

Parsons, R. D., \& Hinton, J. A. 2014, Astropart. Phys., 56, 26

Parsons, R., Murach, T., \& Gajdus, M. 2015, Int. Cosmic Ray Conf., 34, 826

Petropoulou, M., \& Dimitrakoudis, S. 2015, MNRAS, 452, 1303

Poutanen, J., \& Stern, B. 2010, ApJ, 717, L118

Punch, M., Akerlof, C. W., Cawley, M. F., et al. 1992, Nature, 358, 477

Pushkarev, A. B., Kovalev, Y. Y., Lister, M. L., \& Savolainen, T. 2009, A\&A, 507, L33

Pushkarev, A. B., Kovalev, Y. Y., Lister, M. L., \& Savolainen, T. 2017, MNRAS, 468,4992

Ramakrishnan, V., Hovatta, T., Nieppola, E., et al. 2015, MNRAS, 452, 1280

Rani, B., Lott, B., Krichbaum, T. P., Fuhrmann, L., \& Zensus, J. A. 2013, A\&A, 557, A71

Reimer, A. 2007, ApJ, 665, 1023

Reimer, A. 2012, J. Phys. Conf. Ser., 355, 012011

Rolke, W. A., López, A. M., \& Conrad, J. 2005, Nucl. Instrum. Methods Phys. Res. A, 551, 493

Roming, P. W. A., Kennedy, T. E., Mason, K. O., et al. 2005, Space Sci. Rev., 120, 95

Roming, P. W. A., Koch, T. S., Oates, S. R., et al. 2009, ApJ, 690, 163

Salamon, M. H., \& Stecker, F. W. 1998, ApJ, 493, 547

Sambruna, R. M., Maraschi, L., \& Urry, C. M. 1996, ApJ, 463, 444

Schlafly, E. F., \& Finkbeiner, D. P. 2011, ApJ, 737, 103

Schmidt, G. D., Stockman, H. S., \& Smith, P. S. 1992, ApJ, 398, L57

Shappee, B. J., Prieto, J. L., Grupe, D., et al. 2014, ApJ, 788, 48

Sikora, M., Begelman, M. C., \& Rees, M. J. 1994, ApJ, 421, 153

Sikora, M., Stawarz, Ł., Moderski, R., Nalewajko, K., \& Madejski, G. M. 2009 ApJ, 704, 38

Sitko, M. L., Schmidt, G. D., \& Stein, W. A. 1985, ApJS, 59, 323

Smith, P. S. 1996, in Blazar Continuum Variability, eds. H. R. Miller, J. R. Webb, \& J. C. Noble, ASP Conf. Ser., 110, 135

Smith, P. S., Montiel, E., Rightley, S., et al. 2009, ArXiv e-prints [arXiv:0912.3621]

Tavecchio, F., \& Ghisellini, G. 2012, ArXiv e-prints [arXiv:1209.2291]

Tavecchio, F., Ghisellini, G., Bonnoli, G., \& Ghirlanda, G. 2010, MNRAS, 405, L94

Telfer, R. C., Zheng, W., Kriss, G. A., \& Davidsen, A. F. 2002, ApJ, 565, 773

Urry, C. M., \& Padovani, P. 1995, PASP, 107, 803

Vaughan, S., Edelson, R., Warwick, R. S., \& Uttley, P. 2003, MNRAS, 345, 1271

Wakely, S. P., \& Horan, D. 2008, Int. Cosmic Ray Conf., 3, 1341

Wandel, A. 1991, A\&A, 241, 5

Weekes, T. C., Cawley, M. F., Fegan, D. J., et al. 1989, ApJ, 342, 379

Woo, J.-H., \& Urry, C. M. 2002, ApJ, 579, 530

Wright, S. C., McHardy, I. M., \& Abraham, R. G. 1998, MNRAS, 295, 799

Yan, D., Zeng, H., \& Zhang, L. 2012, PASJ, 64, 80

Zdziarski, A. A., \& Böttcher, M. 2015, MNRAS, 450, L21

1 Centre for Space Research, North-West University, Potchefstroom 2520, South Africa

2 Laboratoire Leprince-Ringuet, Ecole Polytechnique, CNRS/IN2P3, 91128 Palaiseau, France

3 Max-Planck-Institut für Kernphysik, PO Box 103980, 69029 Heidelberg, Germany

4 Dublin Institute for Advanced Studies, 31 Fitzwilliam Place, Dublin 2, Ireland

5 High Energy Astrophysics Laboratory, RAU, 123 Hovsep Emin St, Yerevan 0051, Armenia

6 Aix Marseille Université, CNRS/IN2P3, CPPM, Marseille, France

7 Department of Physics, Rikkyo University, 3-34-1 Nishi-Ikebukuro, Toshima-ku, Tokyo 171-8501, Japan

8 Laboratoire d'Annecy de Physique des Particules, Univ. Grenoble Alpes, Univ. Savoie Mont Blanc, CNRS, LAPP, 74000 Annecy, France

9 IRFU, CEA, Université Paris-Saclay, 91191 Gif-sur-Yvette, France

10 University of Namibia, Department of Physics, Private Bag 13301, Windhoek 12010, Namibia

11 DESY, 15738 Zeuthen, Germany

12 Department of Physics and Electrical Engineering, Linnaeus University, 35195 Växjö, Sweden
13 School of Physical Sciences, University of Adelaide, Adelaide 5005, Australia

14 LUTH, Observatoire de Paris, PSL Research University, CNRS, Université Paris Diderot, 5 Place Jules Janssen, 92190 Meudon, France

15 Sorbonne Université, Université Paris Diderot, Sorbonne Paris Cité, CNRS/IN2P3, Laboratoire de Physique Nucléaire et de Hautes Energies, LPNHE, 4 Place Jussieu, 75252 Paris, France

16 Laboratoire Univers et Particules de Montpellier, Université Montpellier, CNRS/IN2P3, CC 72, Place Eugène Bataillon, 34095 Montpellier Cedex 5, France

17 GRAPPA, Anton Pannekoek Institute for Astronomy, University of Amsterdam, Science Park 904, 1098 Amsterdam, The Netherlands

18 Friedrich-Alexander-Universität Erlangen-Nürnberg, Erlangen Centre for Astroparticle Physics, Erwin-Rommel-Str. 1, 91058 Erlangen, Germany

19 Astronomical Observatory, The University of Warsaw, Al. Ujazdowskie 4, 00-478 Warsaw, Poland

20 Institut für Astronomie und Astrophysik, Universität Tübingen, Sand 1, 72076 Tübingen, Germany

21 Instytut Fizyki Ja̧drowej PAN, ul. Radzikowskiego 152, 31-342 Kraków, Poland

22 School of Physics, University of the Witwatersrand, 1 Jan Smuts Avenue, Braamfontein, Johannesburg 2050, South Africa

23 Obserwatorium Astronomiczne, Uniwersytet Jagielloński, ul. Orla 171, 30-244 Kraków, Poland

24 Université Bordeaux, CNRS/IN2P3, Centre d'Études Nucléaires de Bordeaux Gradignan, 33175 Gradignan, France

25 Universität Hamburg, Institut für Experimentalphysik, Luruper Chaussee 149, D 22761 Hamburg, Germany

26 APC, AstroParticule et Cosmologie, Université Paris Diderot, CNRS/IN2P3, CEA/Irfu, Observatoire de Paris, Sorbonne Paris Cité, 10, rue Alice Domon et Léonie Duquet, 75205 Paris Cedex 13, France

27 Nicolaus Copernicus Astronomical Center, Polish Academy of Sciences, ul. Bartycka 18, 00-716 Warsaw, Poland

28 Institut für Physik und Astronomie, Universität Potsdam, KarlLiebknecht-Strasse 24/25, 14476 Potsdam, Germany

29 Landessternwarte, Universität Heidelberg, Königstuhl 69117, Heidelberg, Germany

30 Univ. Grenoble Alpes, CNRS, IPAG, 38000 Grenoble, France

31 Institut für Physik, Humboldt-Universität zu Berlin, Newtonstr. 15, D 12489 Berlin, Germany

32 Institut für Astro-und Teilchenphysik, Leopold-FranzensUniversität Innsbruck, 6020 Innsbruck, Austria

33 Centre for Astronomy, Faculty of Physics, Astronomy and Informatics, Nicolaus Copernicus University, Grudziadzka 5, 87-100 Torun, Poland

34 Kavli Institute for the Physics and Mathematics of the Universe (Kavli IPMU), The University of Tokyo Institutes for Advanced Study (UTIAS), The University of Tokyo, 5-1-5 Kashiwa-no-Ha, Kashiwa, Chiba 277-8583, Japan

35 Department of Physics, University of the Free State, PO Box 339, Bloemfontein 9300, South Africa

36 Department of Physics and Astronomy, The University of Leicester, University Road, Leicester LE1 7RH, UK

37 RIKEN, 2-1 Hirosawa, Wako, Saitama 351-0198, Japan

38 Department of Physics, The University of Tokyo, 7-3-1 Hongo, Bunkyo-ku, Tokyo 113-0033, Japan

39 Yerevan Physics Institute, 2 Alikhanian Brothers St., 375036 Yerevan, Armenia

40 Institut für Theoretische Physik, Lehrstuhl IV: Weltraum und Astrophysik, Ruhr-Universität Bochum, 44780 Bochum, Germany

41 Steward Observatory, University of Arizona, Tucson, AZ 85721, USA

42 Now at Institut de Ciències del Cosmos (ICC UB), Universitat de Barcelona (IEEC-UB), Martí Franquès 1, 08028 Barcelona, Spain

43 Now at Physik Institut, Universität Zürich, Winterthurerstrasse 190 , 8057 Zürich, Switzerland 